\title{
ACLIMATACIÓN AL ESTRÉS HÍDRICO DE PLANTAS DE UVA DE MESA CV. CRIMSON SEEDLESS CULTIVADAS EN MACETA
}

\author{
Conesa, M.R. (1), de la Rosa, J.M. (2), Montemurro, L. (3), García, M. (4), Domingo, R. (5), \\ Pérez-Pastor, A. (6)
}

\begin{abstract}
1,2,3,4 Becario de Investigación, Universidad Politécnica de Cartagena, Departamento de Producción Vegetal, Paseo Alfonso XIII, 48. 30203. (Cartagena), charo.conesa@upct.es, josemdlrs@hotmail.com, luca.montemurro@hotmail.it, manuel.garcia@upct.es 5,6 Dr. Profesor, Universidad Politécnica de Cartagena, Departamento de Producción Vegetal, Paseo Alfonso XIII, 48. 30203. (Cartagena), rafael.domingo@upct.es, alex.perezpastor@upct.es
\end{abstract}

\section{Resumen}

El objetivo de este estudio fue evaluar la capacidad de adaptación al estrés hídrico de uva de mesa cv. Crimson Seedless $x$ Paulsen 1103 cultivadas en maceta bajo invernadero. Se aplicaron 5 tratamientos de riego: (i) CTL-1 y CTL-2; regados diariamente a capacidad de campo; (ii) RD, regado al $50 \%$ del CTL-1; (iii) $P R D_{\mathrm{FIX}}$, regado al $50 \%$ del CTL-

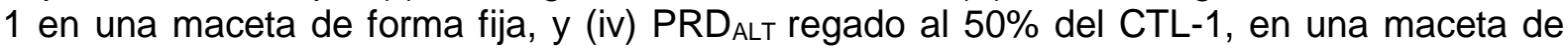
forma alterna. Transcurridos 30 días desde su aplicación, se suprimió el riego durante 7 días a excepción de CTL-1. Posteriormente se reanudo el riego, y todas las plantas fueron tratadas como CTL-1 durante 7 días. El comportamiento estomático de Crimson se caracterizó como isohídrico, dado que reducciones de $\mathrm{g}_{\mathrm{s}}$ por debajo de $40 \mathrm{mmol} \mathrm{m} \mathrm{m} \mathrm{s}^{-1}$, mantuvieron valores de $\Psi_{\mathrm{t} \text {,md }}$ dentro de un rango constante. Las condiciones de déficit hídrico afectaron severamente a las plantas de los tratamientos $P R D_{\mathrm{FIX}}$ y $C T L-2$, dado que, independientemente de la recuperación observada en los valores de $g_{s}$ y $A_{c 02}$, los daños generados sobre su crecimiento vegetativo resultaron ser irreversibles. $P_{R D_{A L T}}$ y $R D$ pueden ser utilizados con fines de programación de riegos.

\section{1.- Introducción}

La demanda internacional de uva de mesa apirena, se ha incrementado en los últimos años. Sin embargo, su cultivo en zonas mediterráneas obliga a adoptar nuevas estrategias de riego que permitan reducir el consumo de agua sin afectar negativamente a su producción (Conesa et al., 2014). El conocimiento de los mecanismos de tolerancia al déficit y la capacidad de los cultivos para experimentar aclimataciones, resulta de gran interés en la confección de estos programas. Por tanto, para su correcta aplicación, resulta necesario conocer la respuesta fisiológica de las plantas al déficit hídrico. En general, la vid es considerada una especie que evita el estrés hídrico con un control estomático estricto (Schultz, 2003, Chaves et al., 2010). Sin embargo, algunas variedades han mostrado un control más estricto que otras, lo que ha llevado a clasificar las variedades de vides como isohídricas o anisohídricas (Tardieu y Simonneau, 1998; Lovisolo et al., 2010). Un comportamiento isohídrico proviene de una fuerte regulación estomática de la transpiración, lo que produce un potencial de hoja casi constante. Al contrario, especies anhisohídricas, poseen un menor control estomático de la transpiración, produciendo una reducción del potencial hídrico foliar de acuerdo al estado energético del agua en el suelo y demanda climática (Conesa et al., 2013).

Numerosas investigaciones han resaltado la importancia que ejerce el nivel de estrés hídrico en la hoja sobre las propiedades estructurales e intercambio gaseoso de la vid (Chaves et al., 2010; Romero et al., 2014). Sin embargo, en uva de mesa, el impacto que 
ejerce la aclimatación de las plantas al déficit hídrico en este intercambio, está poco documentado. El objetivo de este trabajo fue comprobar la capacidad de adaptación (endurecimiento) y tolerancia a la sequía de la uva de mesa ante diferentes niveles de estrés hídrico.

\section{2.- Materiales y métodos}

\section{Condiciones experimentales}

El ensayo se realizó bajo invernadero en la Estación Experimental Agroalimentaria 'Tomás Ferro' de la ETSIA-UPCT, situada en la Palma, (Murcia), durante el verano de 2014. Los plantones de uva de mesa cv. Crimson Seedless fueron injertados en vivero sobre patrón Paulsen 1103 y trasplantados a macetas de 5 litros sobre sustrato de fibra de coco. Se seleccionaron 9 plantas por tratamiento de similar desarrollo y aspecto $(75 \mathrm{~cm}$ de altura y $1 \mathrm{~cm}$ de diámetro de tronco). Al inicio del experimento, el sistema radicular de todas las plantas se dividió en dos mitades (cada mitad en una maceta), a fin de mantener similares condiciones experimentales (Fig. 1). La radiación fotosintéticamente activa, temperatura del aire y humedad relativa (RFA, $T_{a}$ y $H R$ ) fueron registradas en una estación climática instalada dentro del invernadero. A partir de $T_{a}$ y HR se calculó el DPV. El sistema de riego consistió en una hilera de plantas con un emisor por maceta de $2 \mathrm{~L} \mathrm{~h}^{-1}(2$ emisores planta-1).

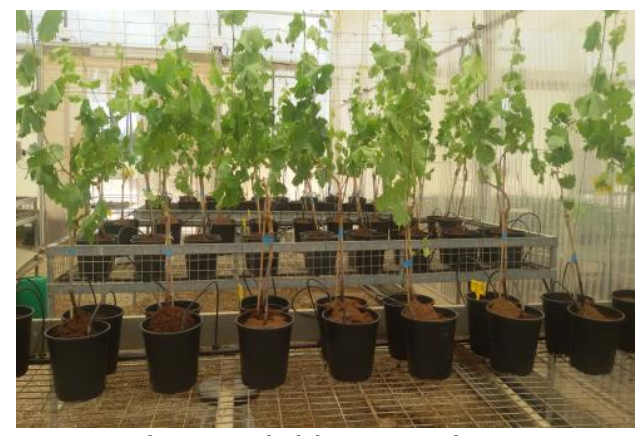

Figura 1. Distribución de macetas dentro del invernadero

\section{Periodos de riego y tratamientos}

Todas las plantas se regaron diariamente durante 30 días con idénticas dosis de agua y solución nutritiva vegetativa. Posteriormente, se establecieron 5 tratamientos de riego de acuerdo a un diseño experimental al azar de 3 repeticiones por tratamiento: (i) CTL1 y CTL-2, ambos regados diariamente a condiciones de humedad de suelo correspondiente a capacidad de campo ( $\Theta_{\mathrm{cc}} \approx 28 \%$ ), excepto las plantas del tratamiento CTL-2 que fueron sometidas al período de estrés y posterior recuperación; (ii) Riego deficitario (RD), dosis equivalente al $50 \%$ del CTL-1; (iii) Riego fijo por desecación parcial de raíces ( $P R D_{\mathrm{FIX}}$ ), dosis equivalente al $50 \%$ del CTL-1, aplicado en una maceta de forma fija. (iv) Riego alterno por desecación parcial de raíces $\left(\mathrm{PRD}_{\mathrm{ALT}}\right)$, dosis equivalente al $50 \%$ del CTL-1, aplicado en una maceta. La alternancia se realizó, cuando el contenido de agua en el suelo $\left(\Theta_{v}\right)$ de la maceta sin riego se reducía al $12 \%$ (correspondiente al punto de marchitez). Estos tratamientos se aplicaron durante 1 mes (periodo de endurecimiento) e inmediatamente después, se suprimió el riego durante 7 días en todos los tratamientos (periodo de estrés), a excepción del tratamiento CTL-1, que se mantuvo regado en las condiciones iniciales. Finalizado el periodo de estrés, todas las plantas fueron tratadas como las plantas CTL-1, con riego diario (periodo de recuperación).

\section{Medidas realizadas}


El estado hídrico de las plantas fue evaluado en 6 hojas sanas por tratamiento de riego, a partir del potencial hídrico de tallo a mediodía $\left(\Psi_{\mathrm{t}, \mathrm{md}}\right.$, entre las 12:00 y las 13.30 hora solar) y antes del alba ( $\Psi_{\mathrm{pd}}$, entre las 5:00 y 6:30 hora solar) con cámara de presión (Soil moisture Equipment Corp., Model 3000), siguiendo las recomendaciones de Hsiao (1990). El contenido volumétrico de agua en el suelo $\left(\Theta_{v}\right)$ fue determinado a partir de sondas GS3 (Decagon Devices Inc.) colocadas a $20 \mathrm{~cm}$ de profundidad en 3 plantas por tratamiento. Los parámetros de intercambio gaseoso (i) conductancia estomática máxima $\left(\mathrm{g}_{\mathrm{sm}}\right)$, (ii) asimilación neta de $\mathrm{CO}_{2},\left(\mathrm{~A}_{\mathrm{Im}}\right)$ y (iii) eficiencia instantánea de uso del agua (WUE, $\mathrm{g} \mathrm{CO}_{2} / \mathrm{g}$ $\mathrm{H}_{2} \mathrm{O}$ transpirada) se determinaron con un medidor portátil CIRAS-2 en condiciones de saturación (radiación fotosintéticamente activa, $\mathrm{PAR} \approx 1500 \mathrm{mmol} \mathrm{m}^{-2} \mathrm{~s}^{-1}$, concentración de $\mathrm{CO}_{2} \approx 380 \mathrm{ppm}$ y temperatura foliar $\approx 30^{\circ} \mathrm{C}$ ). La temperatura del dosel vegetal se determinó con una pistola de infrarrojos digital (Model GM320) en las mismas hojas donde se obtuvieron los parámetros de intercambio gaseoso. A partir de los valores de $T_{f}$ y $T_{a}$, se calculó el índice de estrés hídrico (CWSI) aplicando la fórmula de Idso et al. (1981):

$$
\mathrm{CWSI}=\frac{(\mathrm{dT}-\mathrm{dT} l)}{\mathrm{dTu}-\mathrm{dT} l}
$$

(Ecuación 1)

Donde: dT es la diferencia entre la temperatura del aire ( $\mathrm{Ta}$ ) y de la hoja (Tf) expresado como (Tf-Ta), dTu es el límite superior de la diferencia de temperatura (Tf-Ta), y dTI es el límite inferior de Tf-Ta. Los valores de CSWI varían entre 0 y 1 , donde 0 indicaría condiciones de riego adecuado y 1 condiciones de estrés máximo, y en consecuencia menor transpiración soportada por las plantas.

La epinastía, evaluada durante el periodo de endurecimiento como el cambio en el ángulo de inserción foliar (AIF), fue medida a mediodía solar con un transportador de ángulos transparente, en diez hojas por planta y 3 plantas por tratamiento. Por último al final del periodo de estrés/recuperación se evalúo el crecimiento vegetativo mediante un estudio visual de la calidad de las plantas. Para ello se establecieron 4 categorías, que fueron expresadas en porcentaje: 1) plantas en condiciones ideales, 2) plantas en condiciones aceptables, 3) plantas con daños moderados, 4) plantas con daños severos.

\section{Análisis estadístico}

El análisis de varianza (ANOVA) se realizó con el programa SPSS versión 13.0 para Windows, así como la prueba de comparación múltiple de Duncan, al nivel de confianza del $95 \%$.

\section{3.- Resultados y discusión}

\section{Periodo de endurecimiento}

Los valores medios de DPV a mediodía fueron de $2.78 \mathrm{kPa}$ durante el periodo de endurecimiento, mientras que RFA y Ta promediaron valores de $770 \mathrm{~W} \mathrm{~m}^{-2}$ y $33.1^{\circ} \mathrm{C}$, respectivamente. El tratamiento CTL-1 mantuvo valores de $\Theta_{v}$ próximos a capacidad de campo $(\approx 30 \%)$ a lo largo del experimento, indicando que su suministro hídrico fue adecuado (Conesa et al., 2014). Por su parte, los tratamientos RD, PRD FIX y PRD ALT $_{\text {mostraron valores }}$ medios de $\Theta_{v}$ un 27, 55 y $50 \%$ más bajos que CTL-1 (Fig. 2).

$P R D_{F I X}$, recibió la misma cantidad de agua que $P R D_{A L T}$ y $R D$. Sin embargo, los resultados de $\Theta_{v}$ en PRD $_{\mathrm{FIX}}$ mostraron un importante agotamiento de agua en el suelo, llegando a valores medios de humedad volumétrica de 10.2 y $14.3 \%$, en el lado húmedo y seco, respectivamente. Por tanto, aunque el riego fue el mismo (misma dosis y frecuencia), el agotamiento en el suelo de PRD $D_{\text {FIX }}$ se produjo antes en la zona mojada que en PRD ALT $_{\text {y }}$ 
$\mathrm{RD}$; de forma que, valores de $\Theta_{v}$ a lo largo del día más bajos después del riego, generaron

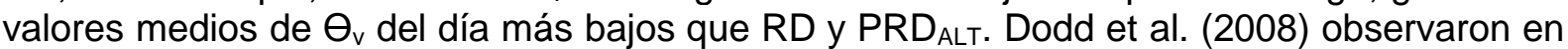
girasol, una reducción de la fracción de flujo de savia cuando se alcanzaron niveles de $\Theta_{v}$ inferiores al $16 \%$ en el lado seco de PRD, limitando así la concentración de ácido abscísico (ABA) que es transportado vía xilema hacia las hojas.

A pesar de las diferencias descritas anteriormente en $\Theta_{v}$, todos los tratamientos deficitarios desarrollaron a nivel de planta, déficit hídricos moderados y similares entre sí, según indican los valores de potencial hídrico foliar antes del alba $\left(\Psi_{\mathrm{pd}} \sim-0.12 \mathrm{MPa}\right)$ (Tabla 1). $\Psi_{\mathrm{t} \text { tm }}$ presentó una tendencia similar a $\Psi_{\mathrm{pd}}$ tal y como se muestra en la correlación encontrada $\left[\Psi_{\mathrm{pd}}=-1.86-0.58 \Psi_{\mathrm{t}, \mathrm{md}} ; \mathrm{r}^{2}=0,67^{\star \star *}\right]$. De forma similar a lo observado para $\Psi_{\mathrm{pd}}$, en todos los tratamientos sometidos a déficit hídrico durante el periodo de endurecimiento, se produjo una reducción drástica del ángulo de inserción foliar (AIF) siendo significativamente más acusada en el tratamiento $\mathrm{PRD}_{\mathrm{FIX}}$ (Fig. 3).

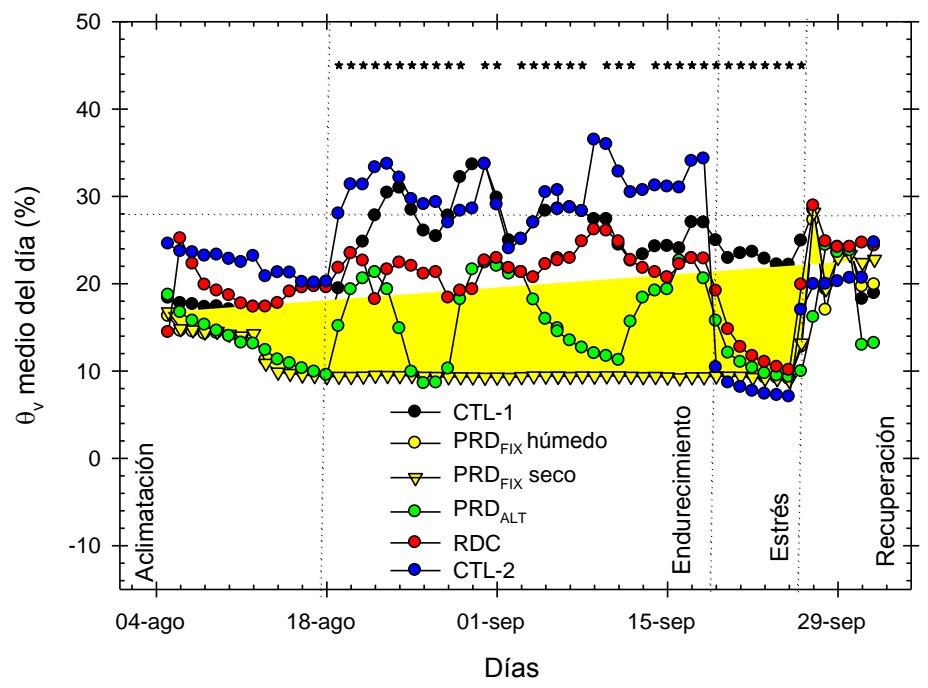

Figura 2. Valores medios de contenido volumétrico de agua en el suelo $\left(\Theta_{\mathrm{v}}\right)$. Cada punto corresponde a la media de de 3 sondas por tratamiento. Notar que, el tratamiento $\mathrm{PRD}_{\mathrm{FIX}}$ estaba monitorizado con 3 sondas tanto en el lado seco, como en el lado húmedo. Las líneas de puntos delimitan los periodos de riego. Los asteriscos indican diferencias significativas de los tratamientos deficitarios respecto a CTL-1.

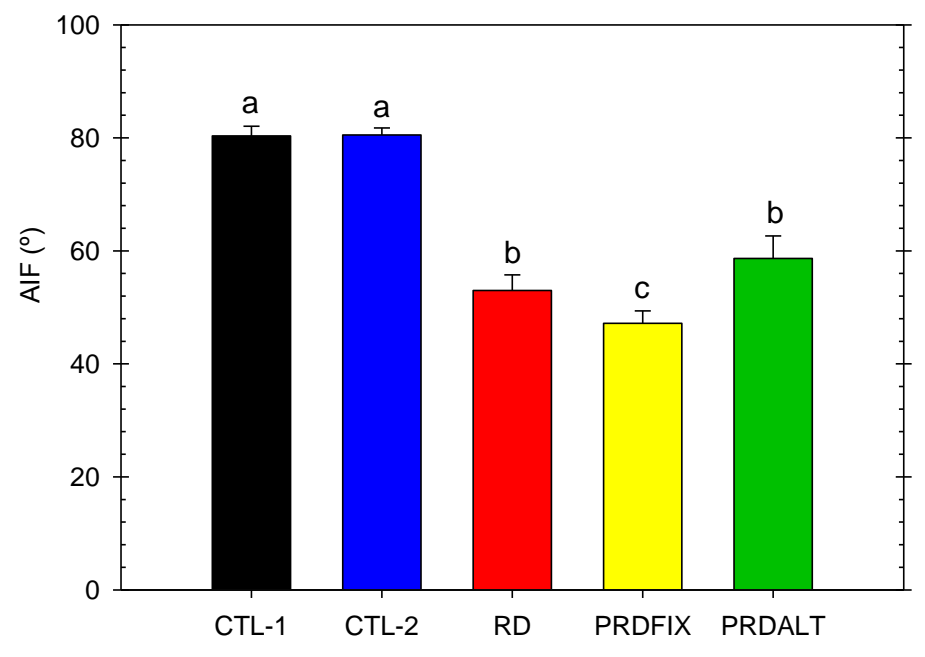

Figura 3. Valores promedio del ángulo de inserción foliar (AIF) durante el periodo de endurecimiento en cada uno de los tratamientos de riego ensayados. Cada barra representa la media \pm error estándar de 30 hojas por tratamiento. 
Tabla 1. Valores medios de los parámetros medidos del potencial hídrico de tallo a mediodía $\left(\Psi_{t, m d}\right)$, potencial hídrico al alba $\left(\Psi_{\mathrm{pd}}\right)$, asimilación neta de $\mathrm{CO}_{2}\left(\mathrm{~A}_{\mathrm{co} 2}\right)$, conductancia estomática $\left(g_{s}\right)$, eficiencia instantánea de uso del agua (WUE) y diferencia de temperatura entre la hoja y el aire (Tf-Ta) durante el periodo de endurecimiento (End.), estrés (E) y recuperación $(\mathrm{R})$.

\begin{tabular}{|c|c|c|c|c|c|c|c|c|c|}
\hline & & \multirow{2}{*}{\multicolumn{3}{|c|}{$\mathbf{R}$}} & \multirow{2}{*}{\multicolumn{3}{|c|}{ End. }} & \\
\hline & & & & & & & & $E$ & $\mathbf{R}$ \\
\hline \multirow{6}{*}{$\boldsymbol{\Psi}_{\mathrm{t}, \mathrm{md}}$} & CTL-1 & $-0.62 a^{z}$ & -0.61 a & -0.69 & \multirow{5}{*}{$\Psi_{\mathrm{pd}}$} & CTL-1 & $-0.04 a$ & $-0.10 a$ & -0.12 \\
\hline & CTL-2 & $-0.62 a$ & $-1.55 b c$ & -0.65 & & CTL-2 & $-0.04 a$ & $-1.15 b c$ & -0.50 \\
\hline & RD & $-0.81 b$ & $-1.46 b$ & -0.71 & & RD & $-0.12 b$ & $-1.02 b$ & -0.37 \\
\hline & PRD $_{\text {ALT }}$ & $-0.74 b$ & $-1.43 b$ & -0.74 & & PRD $_{\text {ALT }}$ & $-0.13 b$ & $-1.13 b$ & -0.57 \\
\hline & $\mathrm{PRD}_{\mathrm{FIX}}$ & $-0.74 b$ & $-1.77 c$ & -0.67 & & PRD $_{\text {FIX }}$ & $-0.13 b$ & $-1.52 c$ & -0.47 \\
\hline & ANOVA & 0.022 & 0.027 & N.S & & ANOVA & 0.019 & 0.035 & N.S \\
\hline \multirow{6}{*}{$A_{\mathrm{CO} 2}$} & CTL-1 & $5.50 \mathrm{a}$ & $6.38 \mathrm{a}$ & 8.16 & \multirow{5}{*}{$g_{s}$} & CTL-1 & $101.65 \mathrm{a}$ & $124.42 \mathrm{a}$ & 163.75 \\
\hline & CTL-2 & $5.08 \mathrm{a}$ & $2.10 \mathrm{~b}$ & 5.1 & & CTL-2 & $91.32 \mathrm{ab}$ & $60.83 \mathrm{~b}$ & 148.33 \\
\hline & RD & $4.99 a b$ & $1.55 \mathrm{~b}$ & 5.33 & & RD & $77.00 \mathrm{~b}$ & $45.83 \mathrm{~b}$ & 119 \\
\hline & PRD $_{\text {ALT }}$ & $4.23 a b$ & $1.28 \mathrm{~b}$ & 5.63 & & $\mathrm{PRD}_{\mathrm{ALT}}$ & 71.47 b & $41.08 \mathrm{~b}$ & 125.92 \\
\hline & PRD $_{\mathrm{FIX}}$ & $3.49 \mathrm{~b}$ & $1.76 \mathrm{~b}$ & 5.57 & & PRD $_{\text {FIX }}$ & 74.67 b & $60.08 b$ & 158.42 \\
\hline & ANOVA & 0.031 & 0.027 & N.S & & ANOVA & 0.019 & 0.035 & N.S \\
\hline \multirow{6}{*}{ WUE } & CTL-1 & 60.05 & 57.7 & 51.17 & \multirow{5}{*}{ Tf-Ta } & CTL-1 & 0.15 & -0.65 & -1.09 \\
\hline & CTL-2 & 62.73 & 47.11 & 37.6 & & CTL-2 & -0.14 & 1.43 & -0.13 \\
\hline & RD & 67.17 & 40.73 & 58.26 & & RD & 1.51 & 1.98 & 0.85 \\
\hline & PRD $_{\text {ALT }}$ & 51.2 & 40.62 & 44.67 & & PRD $_{\text {ALT }}$ & 2.02 & 2.35 & 0.9 \\
\hline & PRD $_{\text {FIX }}$ & 62.04 & 44.32 & 42.39 & & PRD $_{\mathrm{FIX}}$ & 2.29 & 2.28 & 0.99 \\
\hline & ANOVA & N.S & N.S & N.S & & ANOVA & 0.038 & NS & N.S \\
\hline
\end{tabular}

$\mathrm{z}$ Letras diferentes en cada columna indican diferencias significativas entre tratamientos según test de Duncan $(\mathrm{P}<0.05)$. N.S indica diferencias no significativas entre tratamientos.

Durante el periodo de endurecimiento, $g_{s m}$ se redujo significativamente por el efecto del estrés hídrico en los tratamientos deficitarios (RD, $P R D_{\mathrm{FIX}}$ y $P R D_{\mathrm{ALT}}$ ) respecto a $\mathrm{CTL}-1$ (Tabla 1). Sin embargo, los niveles de $A_{\mathrm{CO} 2}$ sólo disminuyeron significativamente en $\mathrm{PRD}_{\mathrm{FIX}}$, confirmando que ante niveles de déficit moderado, las plantas limitan las perdidas por transpiración, manteniendo su productividad foliar (Ruiz-Sánchez et al. 2000). Por otra parte, los niveles medios de la diferencia de temperatura entre la hoja y el aire (Tf-Ta), mostraron como las vides control mantuvieron sus hojas unos $\sim 3 \stackrel{\circ}{\circ}$ por debajo de la temperatura ambiental ( $\mathrm{Ta}$ ), lo que indica una alta capacidad de refrigeración, que proporciona niveles adecuados de transpiración (Ruiz-Sánchez et al. 2000).

\section{Periodo de estrés/recuperación}

Los valores medios de DPV a mediodía fueron de $2.06 \mathrm{kPa}$ durante el periodo de estrés/recuperación, mientras que RFA y Ta promediaron valores de $368,5 \mathrm{~W} \mathrm{~m}^{-2}$ y $29.2^{\circ} \mathrm{C}$ respectivamente. Tras 7 días de supresión del riego (a excepción de CTL-1, que se mantuvo regado en las condiciones iniciales), el nivel de $\theta_{v}$ fue muy bajo y similar en todos los tratamientos (en torno a $10 \%$, Figura 2). Atendiendo a los niveles de $\Psi_{\mathrm{pd}}$, las plantas del 
tratamiento CTL-2 y de $P R D_{F I X}$, alcanzaron niveles de estrés hídrico significativamente más altos, mientras que RD y PRD presentaron niveles moderados (Tabla 1). Además, se observó un efecto positivo del déficit hídrico sobre WUE, con valores absolutos más elevados en RD (Tabla 1). Las condiciones de estrés hídrico aumentaron la diferencia de temperatura entre la hoja y el aire (Tf-Ta), indicando que dicho valor aumenta progresivamente cuando la humedad del suelo comienza a ser limitante, por lo que puede ser utilizado como índice de estimación del estado hídrico (Bellvert et al., 2015). Asimismo, los valores de $\mathrm{g}_{\mathrm{s}}$ y $\mathrm{A}_{\mathrm{co} 2}$ en los tratamientos deficitarios evaluados, sufrieron una disminución drástica durante la fase de estrés (Tabla 1). En este sentido, CSWI mostró valores más cercanos a la unidad en PRD $D_{\mathrm{FIX}}$, seguidos de PRD $\mathrm{ALT}_{\mathrm{L}}$ y RD. Garrot et al. (1990), observaron en 'Flame Seedless' que bajo condiciones de CSWI $\sim 0.20$ se obtuvo un incremento en el peso de bayas, mientras que para valores de CSWI $~ 0.60$ se observó una fuerte disminución de la producción (Fig. 4).

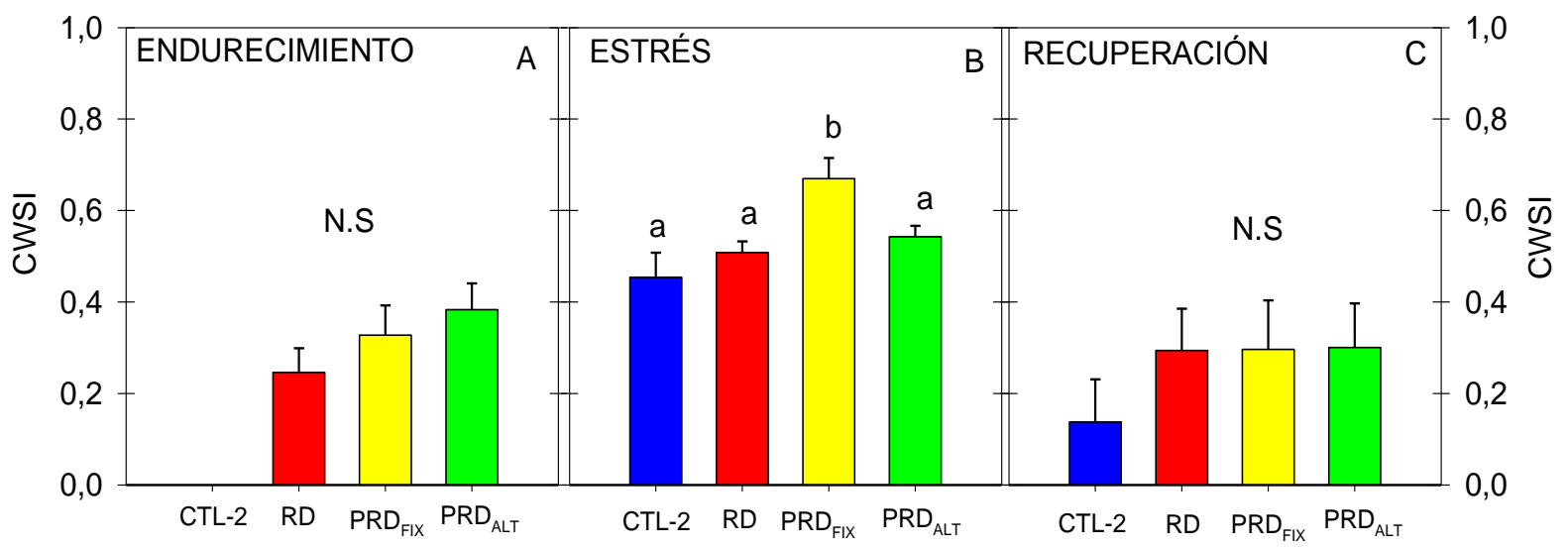

Figura 4. Valores promedio del índice de estrés hídrico del cultivo (CSWI) durante los periodos de: $(A)$, endurecimiento; $(B)$, estrés y $(C)$ recuperación. Cada barra representa la media \pm error estándar de 18 hojas por tratamiento.

Tras la reanudación del riego, la recuperación de los parámetros hídricos estudiados se produjo de forma progresiva, indicando que la influencia negativa del déficit hídrico puede ser reversible (Egea et al. 2012). Cabe señalar, que la recuperación de $g_{s}$ y $A_{c o 2}$ fue ligeramente más rápida en el tratamiento CTL-2 (Fig. 5), debido a que durante el período de estrés, estas plantas sufrieron un mayor grado de defoliación, mecanismo que permite a las plantas reducir la superficie transpirante (mecanismo de evitación a la sequía). Al considerar el conjunto de valores de $g_{\mathrm{s}}$ y $A_{\mathrm{cO} 2}$ se observó una estrecha correlación lineal $\left(\mathrm{A}_{\mathrm{CO} 2}=\right.$ $38.83+9.84 \mathrm{~g}_{\mathrm{s}} ; \mathrm{r}^{2}=0.58^{* * *}$ ). Este hecho sugiere una limitación de la capacidad fotosintética bajo condiciones de estrés hídrico e indica que existe una elevada influencia entre $g_{s}$ y la actividad fotosintética (Egea et al., 2012). Por otra parte, la relación curvilínea encontrada entre $g_{\mathrm{s}}$ y $\Psi_{\mathrm{t}, \mathrm{md}}$ (Fig. 6), mostró que a partir una reducción de $\mathrm{g}_{\mathrm{s}}$ próxima a $40 \mathrm{mmol} \mathrm{m} \mathrm{m}^{-2} \mathrm{~s}^{-1}$, $\Psi_{\mathrm{t}, \mathrm{md}}$ se mantuvo dentro de un rango constante $\left(\Psi_{\mathrm{t}, \mathrm{md}} \sim-1.25 \mathrm{MPa}\right)$. Esta regulación de la transpiración, caracteriza a Crimson como isohídrica, que comparado con el comportamiento de variedades anisohídricas, demuestra mayor tolerancia a la sequía a través de (Lovisolo et al. 2010): (i) aumento de la eficiencia instantánea del uso del agua (WUE), (ii) mayor sensibilidad de los estomas al incremento del DPV, (iii) menor conductancia hidráulica y menor vulnerabilidad a la cavitación. Domec y Johnson, (2012) postularon que los comportamientos isohídricos y anisohídricos en condiciones de riego adecuado o estrés hídrico pueden ser explicados a partir de diferencias en la conductividad hidráulica de la planta $(\mathrm{Kp})$ a medida que varía el DPV. Además, es posible que una combinación de ambas señales (hidráulicas y hormonales), como Kp y el ABA, permita a algunas especies cambiar de comportamiento de isohídrico a anisohídrico y viceversa. Es por tanto necesario señalar, 
que el comportamiento que una variedad puede mostrar durante un experimento está sometido a varias condiciones que modifican este comportamiento, tales como: las condiciones climáticas y edáficas, el déficit hídrico, y el hecho de que la planta haya crecido en el campo o dentro de un invernadero.

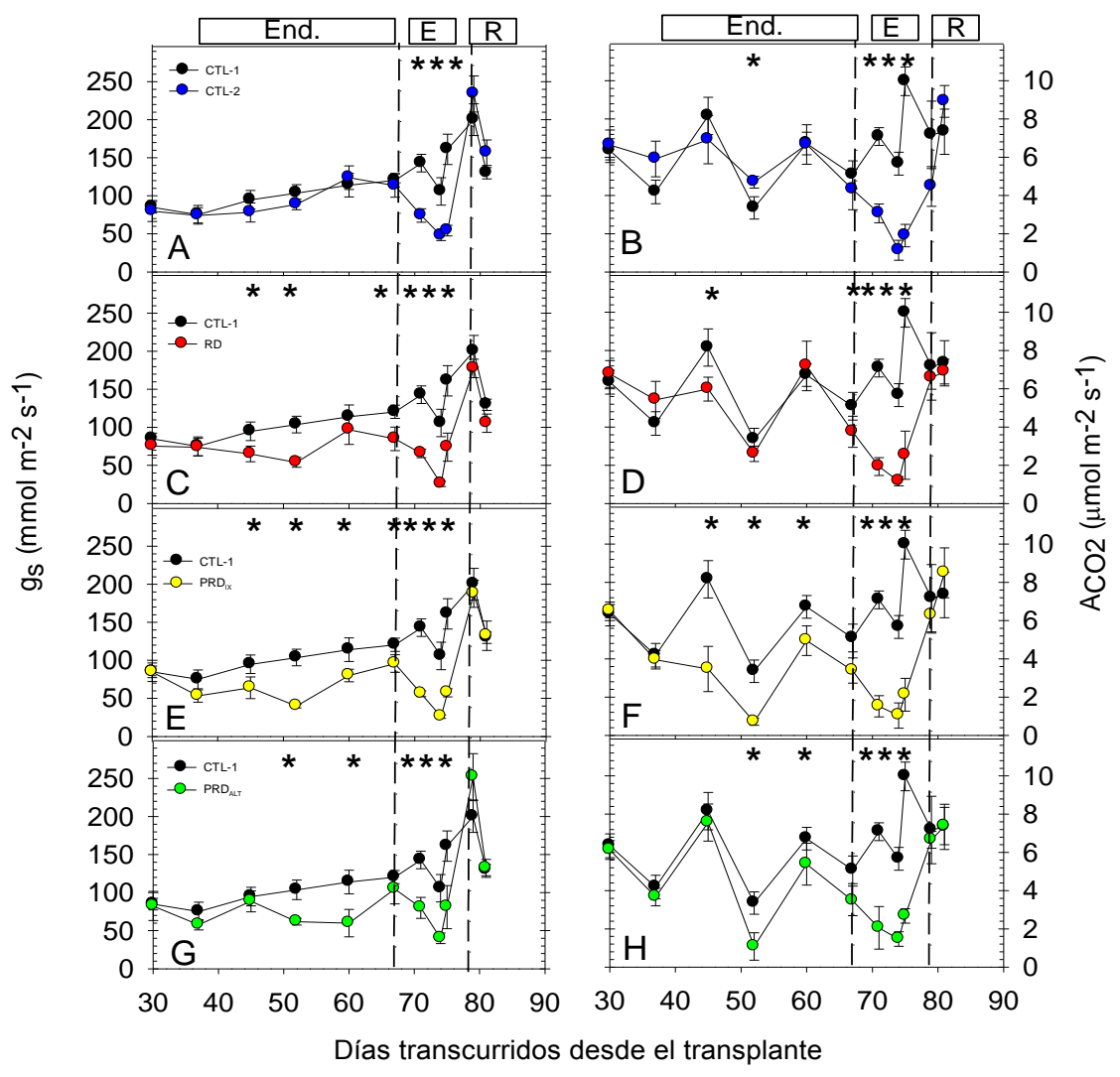

Figura 5. Niveles de conductancia estomática $\left(g_{s}\right)$ y asimilación neta de $\mathrm{CO}_{2}\left(A_{\mathrm{cO} 2}\right)$ durante los periodos de endurecimiento (End.), estrés (E) y recuperación $(R)$ en los distintos tratamientos de riego $\left(C T L-2, R D, P R D_{A L T}\right.$ y $\left.P R D_{F I X}\right)$. Los valores corresponden a la media \pm error estándar de 18 hojas por tratamiento. Los asteriscos indican diferencias significativas respecto a CTL-1.

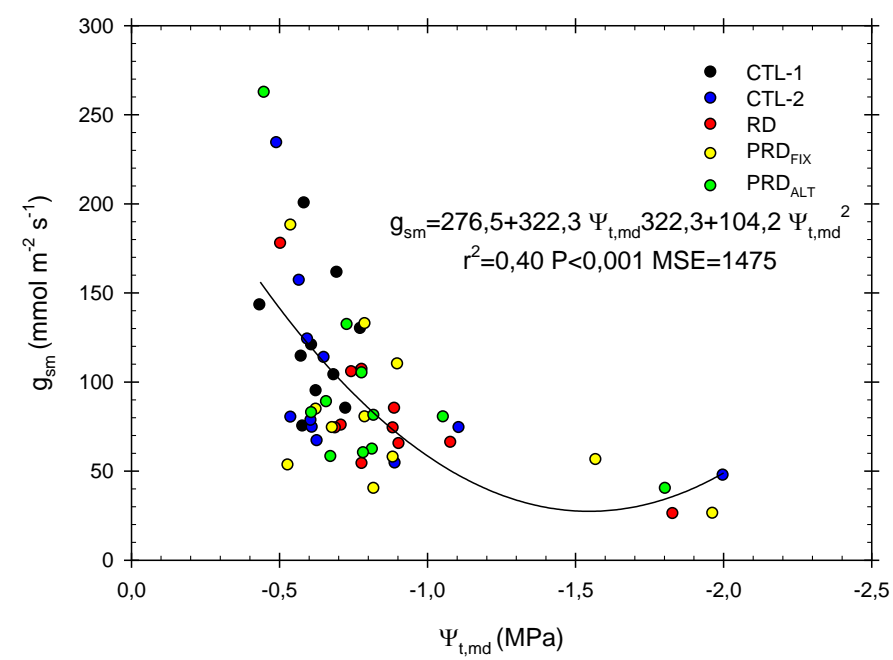

Figura 6. Relación entre potencial hídrico de tallo $\left(\Psi_{\mathrm{t}, \mathrm{md}}\right)$ y la conductancia estomática $\left(\mathrm{g}_{\mathrm{sm}}\right)$ en los distintos tratamientos de riego (CTL-1, CTL-2, RD, PRD ALT y PRD FIX). Cada punto representa la media \pm error estándar de 18 hojas por tratamiento. 
En último lugar, la calidad visual de las plantas evaluadas al final del experimento, mostró daños más severos en los tratamientos $\mathrm{PRD}_{\mathrm{FIX}}$ y CTL-2, inducidos por un mayor grado de defoliación, consecuencia del periodo de supresión del riego (Fig. 7).

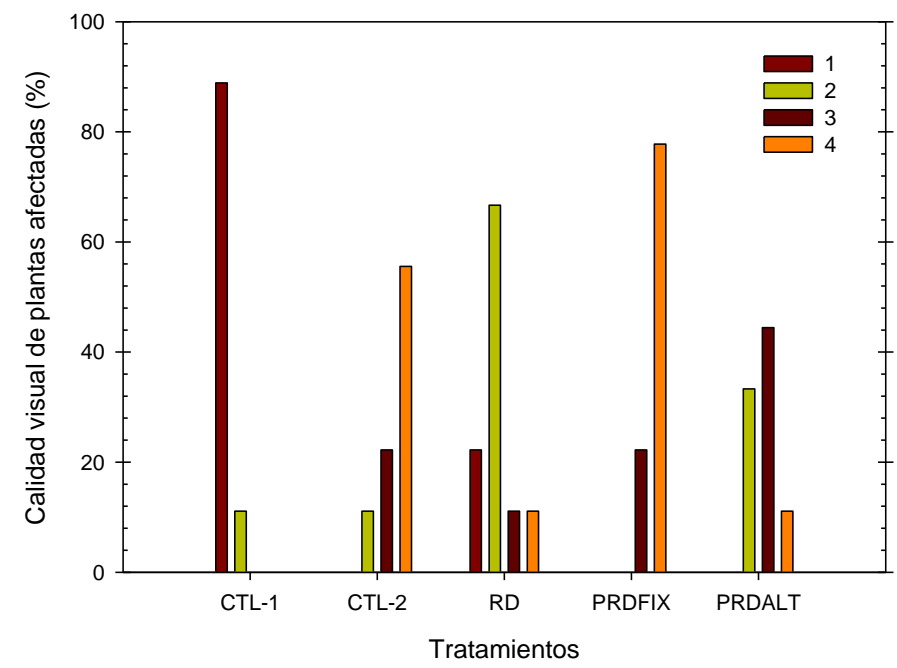

Figura 7. Calidad visual de plantas afectadas al final del experimento en cada tratamiento de riego (siendo: Categoría 1= calidad aceptable $\rightarrow$ Categoría $4=$ daños severos). Cada barra representa la media \pm error estándar de 9 plantas por tratamiento de riego.

\section{4.- Conclusiones y recomendaciones}

El comportamiento estomático de Crimson se clasificó como isohídrico, ya que una reducción de $\mathrm{g}_{\mathrm{s}}$ por debajo de $40 \mathrm{mmol} \mathrm{m}^{-2} \mathrm{~s}^{-1}$, mantuvo valores constantes de $\Psi_{\mathrm{t} \text {,md. }}$ Su respuesta fisiológica, resultó severamente afectada por los tratamientos $\mathrm{PRD}_{\mathrm{FIX}}$ y CTL-2, ya que a pesar de presentar una recuperación de los intercambios gaseosos, los daños generados sobre su aspecto visual y crecimiento vegetativo resultaron ser irreversibles. Sin embargo, las estrategias de $\mathrm{PRD}_{\mathrm{ALT}}$ y $\mathrm{RD}$ mostraron mecanismos de adaptación a la sequía, pudiendo ser utilizadas con fines de programación de riegos.

\section{5.- Agradecimientos}

El presente trabajo se ha realizado con ayuda de fondos del proyecto (AGL-201019201- C04-04) financiado por el ministerio de Educación y Ciencia. Conesa MR, disfrutó de una ayuda del programa de formación de profesorado universitario (FPU).

\section{6.- Bibliografía}

Bellvert, J., Marsal, H., Girona, J., Zarco-Tejada, P.J. (2015). Seasonal evolution of crop water stress index in grapevine varieties determined with high-resolution remote sensing termal imagery. Irrigation Science, 33: 81-93.

Chaves, M.M., Zarrouk, O., Francisco, R., Costa, J.M., Santos, T., Regalado, A.P., Rodrigues, M.L., Lopes, C.M. (2010). Grapevine under deficit irrigation - hints from physiological and molecular data. Annals of Botany, 105: 661-676. 
Conesa, M.R., Pérez-Pastor, A., de la Rosa, J.M., Robles, J.M., Domingo, R., GarcíaSalinas, M.D. (2013). Sensibilidad estomática de 'Crimson Seedless' al déficit hídrico. VI Jornadas de Introducción a la Investigación de la UPCT. pp 61-63.

Conesa, M.R., de la Rosa, J.M., Artés-Hernández, F., Dodd, I.C., Domingo, R., PérezPastor, A. (2014). Long-term impact of deficit irrigation on the physical quality of berries in 'Crimson Seedless' table grapes. Journal of the Science of Food and Agriculture. (In press) DOI 10.1002/jsfa.6983.

Dodd I.C., Egea G., Davies W.J. (2008) Abscisic acid signaling when soil moisture is heterogeneous: decreased photoperiod sap flow from drying roots limits abscisic acid export to the shoots. Plant, Cell and Environment, 31:1263-1274.

Domec, J.C. y Johnson, D.M. (2012). Does homeostasis or disturbance of homeostasis in minimum leaf water potential explain the isohydric versus anisohydric behaviour of Vitis vinifera L. cultivars? Tree physiology, 00: 1-4. Commentary.

Egea, G., González-Real, M.M., Baille, A., Nortes, P.A., Conesa, M.R., RuizSalleres, I. (2012). Effects of water stress on irradiance acclimation of leaf traits in almond trees. Tree Physiology, 32:450-463.

Garrot, D.J., Fangmeier, D.D., Husman, S.H. (1990). Irrigation scheduling using the crop water stress index in Arizona. In: Visions of the Future-Proceedings of the Third National Irrigation Symposium, St. Josephs, MI, ASAE. (pp 281-286).

Idso, S.B., Jackson, R.D., Pinter Jr., P.J., Reginato, R.J., Hatfield, J.L. (1981). Normalizing the stress degree day for environmental variability. Agricultural Meteorology. 24: 45-55.

Lovisolo, C., Perrone, I., Carra, A., Ferrandino, A., Flexas, J., Medrano, H., Schubert, A. (2010). Drought induced changes in development and function of grapevine (Vitis spp.) organs and their hydraulic and non-hydraulic interaction at their whole-plant level: a physiological and molecular update. Functional Plant Biology, 37:98-16.

Romero, P., Pérez-Pérez. J.G., Del Amor, F., Martínez-Cutillas, A., Dodd, I.C., Botía, P. (2014). Partial root zone drying exerts different physiological responses on field-grown grapevine (Vitis vinifera cv. Monastrell) in comparison to regulated deficit irrigation. Functional Plant Biology, 41(11): 1087-110.

Ruiz-Sanchez, M.C., Domingo, R., Torrecillas, A., y Perez-Pastor, A. (2000). Water stress preconditioning to improve drought resistance in young apricot plants. Plant Science, 156: 245-251.

Schultz, H.R. (2003). Differences in hydraulic architecture account for near isohydric and anisohydric bahaviour of two fied grown Vitis vinifera $L$. cultivars during drought. Plant, Cell and Environment, 26(8):1393-1405.

Tardieu, F y Simonneau, T. (1998). Variability among species of stomatal control under fluctuating soil water status and evaporative demand: modelling isohydric and anisohydric behaviors. Journal of Experimental Botany, 49:419-432. 